\title{
HERBAL ACTIVE INGREDIENTS USED IN SKIN COSMETICS
}

\author{
RIYA ARORA ${ }^{1}$, GEETA AGGARWAL ${ }^{2}$, GITIKA ARORA DHINGRA ${ }^{3}$, MANJU NAGPAL ${ }^{1 *}$
}

${ }^{1}$ Chitkara College of Pharmacy, Chitkara University, Punjab, India. ${ }^{2}$ Department of Pharmaceutics, Delhi Pharmaceutical Sciences and Research University, New Delhi, India. ${ }^{3}$ Department of Pharmaceutics, NCRD's Sterling Institute of Pharmacy, Navi Mumbai, Maharashtra, India. Email: manju.nagpal@chitkarauniversity.edu.in

Received: 15 April 2019, Revised and Accepted: 12 July 2019

\begin{abstract}
Human beings are obsessed with looking beautiful. Hence, various beauty products have been in use to look charming and young. Herbal ingredients are popular worldwide for use in cosmetics. The demand for herbal cosmetic is growing in the world market and is an invaluable gift of nature. Herbal skin cosmetics are formulated using different herbal active ingredients, which are further incorporated in cosmetic base to nourish and cure various skin ailments. Herbal cosmetics are natural and are found to be safe to use as compared to chemical-based cosmetics. Herbal formulations have always attracted considerable attention because they are free from all the harmful synthetic chemicals which otherwise may prove to be toxic to the skin. The bioactive ingredients from plants include antioxidants, vitamins, essential oils, tannins, alkaloids, dyes, carbohydrates, and terpenoids, which are used as cosmetics for care of skin, body, and its other parts. The herbal cosmetics which are used on a daily basis, include herbal cream, face wash, lip balm, herbal conditioners, herbal soap, and herbal shampoo. Cosmetics based on herbal ingredients possess desirable physiological activity such as smoothing appearance, healing, enhancing, and conditioning properties. The cosmetic industry is now focusing on this growing segment with an enormous scope of manifold expansion in coming years. Various herbal active ingredients which are particularly used for skincare are being discussed thoroughly in the current review.
\end{abstract}

Keywords: Herbal, Cream, Antioxidant, Anti-aging, Sun blockers, Photoprotection.

(C) 2019 The Authors. Published by Innovare Academic Sciences Pvt Ltd. This is an open access article under the CC BY license (http://creativecommons. org/licenses/by/4. 0/) DOI: http://dx.doi.org/10.22159/ajpcr.2019.v12i9.33620

\section{INTRODUCTION}

Cosmetics can be defined as the materials of various sources, technically compounded substances which can be used to cleanse, nourish, and moisturizes the skin of the face and other parts of the body. They can be used in various forms to alleviate skin problems, modify imperfections, and beautify the skin [1]. The word cosmetic was obtained from "Kosm tikos," a Greek word, means having the influence, arrange, and ability in decorating [2]. Cosmetics are the effective products used broadly all over the world for sustaining and brushing general outlook of the face and other body parts, for example, hand, mouth, finger, hair, lip, and eye. Cosmetics are available in numerous formulations which include creams, face pack, lotions, powder, shampoos, conditioners, and hair oils for radiating, smooth and nourished skin and hair, positively count for an attractive woman and good looking man. In spite of this, cosmetics contain numerous chemical toxins, chemicals, toxic matter, chemical dyes, and their derived products, which can cause human health troubles and side effects directing to countless diseases. Therefore, the allopathic system is not sufficient for healthy benefits and there is a need to opt herbal cosmetics. Nowadays, herbal cosmetics have emerged as the appropriate way out to the ongoing issues [3].

Herbal cosmetics are the composition incorporating phytochemicals from various botanical sources, impacting the skin functions, and allocate nutrients which are beneficial for the healthy and glowing skin or hairs. These phytochemicals of various sources have dual functions, (i) they can be utilized as a cosmetic product for the skincare purpose, and (ii) the botanical components imparting biological activity to the skin and furnish nutrients beneficial for the nourished skin or hair [3].

The need for herbal cosmetics is increasing rapidly in comparison to conventional cosmetics due to lesser side effects. Herbal cosmetics are favorable because they are produced by the herbs and shrubs. Natural components in the herbs do not possess any unwanted or side effect on the skin of the human body but supplement the skin with nourishment and other favorable nutrients [4].
Advantages of herbal cosmetics over conventional or synthetic cosmetics

Eco-friendly

Conventional cosmetics or beauty products exploit petroleum-based components and normally depends on a host of various chemicals for their buildup. These products are usually abrasive substances, such as lead, aluminum, petroleum, and each of them demand huge mining. In fact, the widely used cosmetic products of all antiperspirants and hair dyes, utilize aluminum as a major component, which is tied to Alzheimer's disease and breast cancer development. In spite of this, aluminum mining is accounted for the devastation of wide swaths of rainforests in South America. Adopting aluminum-free antiperspirants is one of the best options to avoid vulnerability to this compound [5].

\section{Lack of harsh chemicals}

Conventional cosmetics may be efficacious for upgrading the outward impression for the time being, but some of the chemicals in conventional cosmetics are often resulted in side effects which may include allergic reaction, irritation in individuals. Moreover, some of the chemicals used in conventional cosmetics can be poisonous for the posterior pituitary gland (endocrine system). Phthalates and parabens are extensively used in conventional cosmetics, and both of these substances have been associated with type II diabetes and cancer [5].

\section{Nutrition rich}

Our skin has the capability to soak up certain substances. However, the stratum corneum (outer layer of epidermis) a preventive barrier is capable to keep hazardous compounds, to several extents, out of the skin. Even though research concludes that the cosmetic products or any skincare products, when used externally (topically), can harm the skin and human body systems and cause serious health hazards. Cocoa butter is an effective organic moisturizer, presenting essential fatty acids for nourished and healthy skin. Herbal cosmetics, mainly liquid foundation, composed of cocoa butter or similar components provide 
healthy and smooth skin. Extracts of apricots, green tea, pomegranate seeds, and grapes offer antioxidant action and may restrain elastase and collagenase, two enzymes responsible for the breakdown of elasticity and integrity of the skin [5]

\section{Fit budget}

Herbal cosmetics are less expensive and moderate when contrasted with conventional products. Estimation of the World Health Organization (WHO) uncovered that around $80 \%$ of the total population depends on herbal products for their health care, on account of antagonistic impacts and higher cost of manufactured items. Because of accessibility and more secure utilization of herbal cosmetics at a lower cost, WHO, presently, recommends and proposes the utilization of natural products on a greater perspective. For this, WHO is sorting out medicinal services projects to benefit such items at lower costs with more esteem and safety [6].

\section{Insurance against early maturing}

A cosmetic made out of natural minerals typically gives a clear measure of sun security. Typically, the skin of the facial area is more slender and substantially more powerless against ultraviolet (UV) beams and skin maturing. In this manner, picking minerals and supplements rich makeup may give smooth, solid, and energetic skin [3]. The sun plays a noteworthy part in rashly maturing the skin [7-9].

There are sufficient quantities of plants and their bioactive concentrates having the potential for enhancing skin and hair conditions. Natural ingredients and their bioactivity can be utilized for the readiness of different formulations of herbal cosmetics. Herbal cosmetics are notable for their hostile to oxidant and defensive activity against pimples, rashes, dermatitis, scabies, warts, skin issues, and other issues [3]. There are numerous spices and herbs that contain active ingredients and compounds, which help to protect the skin [10]

\section{HERBAL COMPONENTS POSSESSING VARIOUS BENEFITS}

Several herbal compounds with their excipient benefits used in various cosmetic formulations are as follows:

\section{Anti-aging treatment}

Two distinct kinds of changes happen in the skin. Changes in the skin coming because of the progression of time alone are called chronological maturing. The other changes resulting because of constant sun frontage refer to the term photoaging. Flattening of the dermal-epidermal junction is the most obvious and reproducible biological feature of the maturing skin. Reduction in the quantity of fibroblasts, diminished levels of collagen and elastin, reflects the general decay of the extracellular matrix (ECM). UV rays stimulate the synthesis of reactive oxygen species (ROS), which has been embroiled in photoaging [11]. Maturing of the skin is essentially identified with diminishments in the levels of Type I collagen, which is the foremost segment of the collagen skin dermis. Type I collagen is known to play out an important function to maintain the skin dermis's structure and the principal part of the ECM [2]. The balance between degradation and synthesis of collagen is the two influencing factors which affect the amount and nature of extracellular collagen. Characteristic phytochemical sources are advantageous to prevent the skin from maturing or wrinkling. Phytochemicals rich extracts such as phenolic acids, saponins, alkaloids, and flavonoids have the property of collagen synthesis and broadly utilized for the advancement of anti-aging topical cosmetics products [7-9].

\section{Ginseng}

Ginseng is incorporated as an anti-maturing component in facial creams. A few therapeutic examinations have demonstrated its viability in defending wrinkles because of ginseng's capacity to support skin-firming collagen [12]. The suppression of UV-induced apoptosis and an increase in the level of type I collagen production results in aging of the skin. The ginsenoside $\mathrm{Rb} 1$ is beneficial to exhibit anti-aging activities and promote youthful skin [13]. The herb additionally goes about as a skin lightning component, giving a more vibrant and younger appearance to the skin [12]. Red ginseng extricate is accepted to have oxidation inhibition, anti-aging, and immuno-stimulatory properties. This is the foremost controlled investigation of red ginseng extract on a human to investigate its impacts on photoaged skin, or damaged skin due to sun introduction. The facial skin of healthy mature volunteers above the age of 40 years was tested before and after the extended duration of treatment using a mixture of red ginseng extract with other herbs [14].

\section{Rosemary}

Rosemary is one of the essential components against aging that can assist the human skin in a few ways. Utilizing rosemary holds the nourished content of the skin, consequently keeping the skin firm and thus prevents the fine lines appear on the facial skin. Rosemary is also helpful for shielding your skin from ecological components and supporting the insurance of collagen in the skin. Counting rosemary as one of the essential components in your healthy skin routine can provide you a smooth and resilient skin [10].

Carrot

Carrot is a fragrant herb procured from the Daucus carota, belonging to the family Apiaceae. Chief components of carrots are carotenes, prevalently $\beta$-carotene. The study revealed that $\beta$-carotene is well known antioxidant and precursor of Vitamin A. Study on $\beta$-carotene confirmed that it is converted into Vitamin A when applied on the skin, as the compounds and factors essential for its transformation to Vitamin A are present on the skin. Topical application of Vitamin A helps to preserve the healthy skin conditions, advances legitimate metabolism of skin and ameliorate mending and roughness, while altogether diminishing the impacts of skin aging. Vitamin A show numerous effects on matured skin:

- It actuates the skin proteins formation, for example, collagen

- The development of a thicker epidermis secured by a superior layer of keratin

- It promotes cell metabolism and cell mitosis and builds skin's flexibility and smoothness

- It is fundamental for basal cell propagation and their appropriate differentiation [15]

Carrot is rich in beta-carotene and Vitamins E, C, B, and D which may impart the skin's immunity and antioxidant action in the fatty region. It also shows antioxidant activity against certain unsaturated fat. Root concentrate of carrot protect the facial skin from free radical harm and aid new cells development and generation. This herbal excipient is amazing for aged and dry skin. Thus, this is an astonishing segment for the items intended to revive drained, damaged, tired, and matured skin. Carrot can be utilized as a part of different cosmetics such as lotion, toner, hair cleanser, and anti-aging products [16,17]

\section{Gingko}

Gingko also known as maidenhair tree, obtained from the Ginkgo biloba, having family Gingkoaceae. Primarily comprises flavonoids and terpenoids that enhance the bloodstream and protect the skin damage Gingko is known for its antioxidant, anti-inflammatory, and anti-aging activity. Therefore, this herb can be utilized as one of the essential components in herbal cosmetics for improving skin quality $[16,18]$. Antioxidant activity of gingko helps to lessen the harm caused by the free radicals in our body, reducing wrinkles, and fine lines maintains the firmness of the skin, and influencing you to look more youthful and healthier [18]. As per the study, researchers found that concentrate of gingko may improve and enhances the collagen production in the skin. According to the International Journal of Cosmetic Science, Gingko could use to protect the human skin from harmful impacts of UV (UVA and UVB) rays exposure $[16,18]$.

\section{Herbal antioxidants}

One of the significant mechanisms involved in dermatological disorder and skin aging is oxidative stress. UV rays from sun exposure are a 
well-known exogenous factor destructive to the skin. The persistent exposure to environmental pollution prompts modifications in the connective tissue because of the development of ROS lipid peroxides, and additionally, chemicals enzymes, which brings about several skin issues. Antioxidants control the formation of free radicals. These radical scavengers inhibit numerous oxidizing chain reactions in the body and provide immunity to the human body. Exogenously generated ROS react with different biomolecules existing in the skin, and play a crucial part in skin issue. In such manner, topical utilization of oxidation inhibiting agent gives an efficient procedure to enhance the endogenous cutaneous framework, prompting a reduction in the UVradiation interceded oxidative harm and counteract oxidative stress intervened diseases. Natural antioxidants or oxidation inhibiting agents prevent the formation of free radicals. Furthermore, the initiation and propagation of oxidative chain reaction of reactive species are inhibited by antioxidants. Thus, antioxidants prevent and repair oxidative damage done by the body's cell stimulated by oxygen $[19,20]$.

\section{Anthocyanins}

Anthocyanins are the natural water-soluble pigments obtained from plants and vegetables [21], providing oxidation inhibiting action through a variety of mechanisms [22].

Japanese study discovered that cyanidins, present in anthocyanins, play an important role in the antioxidant activity. Another examination also demonstrated the oxidation inhibiting action of cyanidins to protect lipids of the cell membrane. In addition, it was discovered that cyanidin has 4 times more powerful oxidation inhibiting property than Vitamin E [23]. Anthocyanins procured from the black raspberry show inhibiting action against advancement and progression of tumor cells. These slowdown the advancement of premalignant cells, prompting the turnover of cell rate, effectively impacting the infection cells to die, and by lessening provocative mediators that begin tumor development [23].

\section{Proanthocyanidin}

Proanthocyanidin (oligomeric proanthocyanidins [OPC]) works as a deoxyribonucleic (DNA) transformation inhibitor. Furthermore, proanthocyanidin prevents elastase, maintains the firmness and the reliability of the skin's elastin. OPC work synergistically with both Vitamins C and E, protect and energize them. Cream formulation of proanthocyanidin exhibits powerful action against the hazardous effects of the sun rays (UV radiations). Less burning sensation was observed with the application of OPC cream before sun exposure [22]. Principal change involved with the aging of the skin is the degradation of collagen filaments and glycosaminoglycans with a subsequent decrease in the skin thickness. Application of antioxidant and OPC by means of the transdermal route in the rat prompted the synthesis of collagen fiber. Moreover, in the fibroblast cell societies, OPC has impelled the level of collagen synthesis [23]. Grape seed proanthocyanidins (GSP) are free radical scavengers and strong oxidation inhibitors. GSP hindered skin tumor development and diminished the skin tumors size in mice when introduced to cancer-causing UV radiation [22].

\section{Carotenoids}

Vitamin E ( $\alpha$-tocopherol) is a potent antioxidant. Acute and chronic sun damage can be prevented by the topical application of this antioxidant. When applied topically, only the natural part of Vitamin E - tocotrienol and alpha-tocopherol - viably diminish skin roughness. It also reduces the extent of facial lines and the intensity of the wrinkles. Topical application of Vitamin E improves hydration of the stratum corneum (outermost layer of the epidermis) and expands its waterrestriction limit. Alpha-tocopherol or Vitamin E diminishes the harmful collagen-destructive enzyme collagenase. Level of this enzyme gets increased in matured skin. Vitamin E is an emollient and free radical scavenger as well. Wheat germ oil (Triticum vulgare) is a rich source of Vitamin E and propose an outstanding antioxidant action in topical anti-aging products or formulations. In addition, it moisturizes and prevents hydration loss from the skin. Hazelnut oil (Corylus avellana) is rich in tocopherols, as sunflower (Helianthus annuus) and sesame (Sesamum indicum) oils. Pumpkin (Cucurbita pepo) seed oil merits more prominent acknowledgment. With a lipid profile comprising elevated amounts of linoleic acid (43-53\%), it also includes two types of antioxidant agents [22].

\section{Retinol}

Retinol is one of the essential ingredients in anti-aging formulations. Retinol's capacity to advance skin-rejuvenation is vital to improving the skin's general appearance. By accelerating turnover of cell rate, retinol may assist in the rejuvenation of the skin. It also helps to improve the facial lines appear. Topical utilization of products containing retinol (i.e., retinol skin cream) shows strong antioxidant activity that assists to kill free radicals that are available in the environment [24].

\section{Herbal sun blockers and photoprotection}

The principal resistance against the sunlight is the generation of melanin which assimilates hazardous UV radiations and subsequently shields skin cells from the undesirable impacts of UV rays. In specific conditions, the quantity of melanin production is not sufficiently adequate to protect or shield the skin [25]. Unlike synthetic marketed sunscreen component, which absorb or block UV radiations, this new group of components aims to ensure protection against the harmful UV rays can do inside the cell [26]. Sun screen items comprises sun blocking agents that can retain or reflect UV rays on the surface of skin and in this way shield the skin from the undesired impacts of UV rays [25].

There are three common examples of natural mineral ingredients used as sun blockers

- Zinc oxide: Provides protection against both UVA and UVB types of rays

- Titanium oxide: Give sun protecting factor 15 protection against the harmful effects of the sun

- Iron oxide: Provides protection against UV rays.

Zinc oxide and titanium oxide are physical sunblocks and provide broad-spectrum UVA and UVB protection. In addition to these, many herbal components provide antioxidant action; Vitamin $\mathrm{C}$ has been confirmed to provide sun protection benefits [5]

Analysts have discovered that some plant oils comprise natural sun blockers, for example, sesame oil resists $30 \%$ of UV beams, though coconut, olive, peanut, and cottonseed oils block out around 20\% [22].

\section{Silymarin}

Silymarin is obtained from the seeds of milk thistle (Silybum marianum). It is a flavonoid compound contains flavonolignans that prevent the superoxide radicals production. Several skin benefits of silymarin are antioxidant, sun damage protection, anti-acne, anti-inflammatory, lightning, and brightening. Silymarin also treats hyperpigmentation. Silymarin has excellent antioxidant property as it scavenges free radicals which are capable of damage cells exposed to toxins. It has been demonstrated that silymarin is ten-folds more potent antioxidant action than Vitamin E. Flavonolignans of silymarin particularly silibinin, have good skin penetration capacity so it can be used as an ideal candidate for topical skin care formulations. Studies showed that silymarin has excellent efficiency in UV protection and skin cancer prevention. Statistical data from several studies have also concluded that it is beneficial for reducing both melasma and rosacea, skin conditions with facial manifestations $[27,28]$.

Silymarin comprises three phytochemicals: Silidianin, silybin, and silicristin [22]. A recent report by the Palacký University demonstrated that topical use of Silymarin was helpful in the prevention of oxidative stress produced by UVA light exposure. Another examination in 2008, which focused on the reasons for UV rays generated stress, demonstrated that CD11b cells are in charge of a significant number of the issues caused by UVB introduction. Topical application of silymarin 
previously or after UV light introduction enormously diminished their negative effects [29].

\section{Ascorbic acid (Vitamin C)}

Vitamin C (L-ascorbic acid) is the body's most essential extracellular and intracellular aqueous phase oxidation inhibitor agent. Ascorbic acid gives numerous advantages to the skin - most fundamentally, it increases the photoprotection and the collagen synthesis. Photoprotection is improved by the anti-inflammatory action of ascorbic acid. Photoprotection enables the skin to recover previous photodamage; the collagen synthesis and MMP-1 inhibition were demonstrated to diminish the facial wrinkles. Its tyrosinase inhibiting and anti-inflammatory activity bring about depigmenting sun lentigines [22]. Vitamin $\mathrm{C}$ is the principal ingredient that alone can provide photoprotection; it works synergistically with Vitamin E (Vitamin E), which potentiates the Vitamin C activity up to 4 times. Hydrophilic Vitamin $C$ assists in the regeneration of Vitamin E, which is a lipophilic oxidation inhibitor agent (antioxidant). Thus, Vitamin E and Vitamin C together secure the lipophilic and hydrophilic section of the cell, respectively. Both the Vitamin $\mathrm{E}$ and Vitamin $\mathrm{C}$ synergistically reduce the harmful UV rays by significantly diminishing the apoptosis of cell and the formation of thymine dimer [30].

Walnut

Extract of walnut is produced using the fresh green shells of English walnut, Juglans Reggie. The aqueous concentrate of walnut has been found to be especially beneficial as a self-tanning sun blocker agent. It mainly comprises naphthol, juglone. Juglone is known to respond with the keratin proteins existing in the skin to make sclerojuglonic compounds. These are providing UV protection activity [22]. Walnut oil is beneficial for aged skin as it fights against wrinkles. Regular topical application of walnut oil lightens the fine lines on the skin and wrinkles to fade away with time [31]

\section{Apigenin}

Apigenin is a broadly distributed herb. Apigenin was observed to be successful in the protection from UVA or UVB rays induced skin carcinogenesis in SKH-1 mice [22]. Apigenin has multiple functions; it acts as an antioxidant, anti-inflammatory, lightning agent, anti-irritant, lightening agent, and antiseptic properties. It is used in a variety of skincare products [32].

\section{Herbal ingredients for skin protection}

The utilization of various bioactive herbs and phytochemicals, obtained from different biological sources accomplish different skincare functions: Care of body skin and as an important component to influence the skin's biological function, giving the supplements to healthy skin. These herbal compounds are rich source of essential oils, vitamins, proteins, antioxidants, terpenoids, and other active components. Different composition of these extracts has a different activity, depending on their nature. There is a remarkable increase of phenolic oxidation inhibitor agent in the most recent decade because of their high ability to scavenge free radicals. Herbal compounds rich in phenolic components can be utilized for skin protection [20].

Phenolics and tocopherols represent 59\% of the oxidation inhibitor action. It is particularly esteemed in the traditions of Central and Eastern Europe and the Middle East for its healthful advantages and is utilized in both the ways either orally or topically for medicinal value. Because of its potency and rich odor, it is just utilized in little extents in topical products. Mineral oil dissolves the secreted sebum from the oil glands, thus helps in skin protection and assists skin evaporation [22]. Herbal excipients are especially incorporated as an essential ingredient in skincare cosmetics because of several desirable properties, for example, anti-inflammatory, antioxidant, and antimicrobial antiseptic properties. The natural components, all in all, or part, have been utilized for different infirmities of the skin [33].
Borage oil

Borage oil is obtained from Borago officinalis. This oil prompt skin cell action and encourage regeneration of the skin. Borage oil is rich in gamma-linoleic acid (GLA), making it helpful in curing the skin problems, especially hypersensitivities, dermatitis, irritation, and inflammation. Borage oil can penetrate through the skin effectively and benefits a wide range of skin, especially dry, mature, dehydrated, or premature aging skin [22].

\section{Evening primrose oil}

Evening primrose oil is obtained from the Oenothera biennis. It is normally yellow in color. This oil has a high level of GLA that advances healthful skin and repair of skin cells. It mitigates skin issues and irritation, making it a great option for individuals with skin inflammation, psoriasis, or any kind of dermatitis. Primrose oil prevents dry skin and untimely maturing of the skin [22].

\section{Avocado oil}

Avocado is also known as Persea Americana. Avocado oil comprises Vitamin E, Vitamin D, $\beta$ - carotene, protein, fatty acid, and lecithin. Avocado oil offers impressive advantages when added to formulations. From avocado oil to botanicals, for example, comfrey and rosemary, these herbal excipients alleviate and secure the skin [22].

\section{Tea tree oil}

Tea tree oil is obtained from Melaleuca alternifolia. This oil is an old native remedy [22]. It consists of strong antifungal and antibacterial components that are known to decrease irritation, which can prompt skin inflammation [34]. It is also a strong disinfectant and antiseptic. It is an essential ingredient of numerous sunscreen products that mitigate sunburn by enhancing blood stream in vessels, conveying supplements to harming skin [22]. A study from the Skin Disease and Leishmaniasis Research Center demonstrated that tea tree oil was powerful for mild to moderate skin inflammation and acne. Tea tree oil is a popular component in cosmetic remover as well [34]

\section{Krameria triandra}

The root extracts of $K$. triandra have great antioxidant and photoprotective potential. Its antioxidant and photoprotective activity has been assessed in various cell models, human keratinocytes cell lines and rat erythrocytes, introduced to chemical and physical (UVB radiation). Root extracts of $K$. triandra remarkably and dose-dependently restrained the loss in the feasibility of cell and the intracellular oxidative harm. Root extract also showed the cytoprotective impact in a more serious model of damage cell: Keratinocytes exposure to higher UVB dosages, which induce a half cell death. Culture of keratinocyte supplemented with $10 \mu \mathrm{g} / \mathrm{ml}$, completely preserve the cell feasibility and more effectively than with green tea and (-) epigallocatechin-3gallate (EGCG). The conclusion of this examination demonstrates the potential utilization of Rhatany extract, standardized in neolignans, as topical radical scavengers and antioxidant against photodamage of skin [22].

\section{Allantoin}

Allantoin is also known as Aldioxia. It is particularly utilized for its antiseptic and anti-inflammatory action. Allantoin is known to produce quick turnover of cell and skin cell generation by eliminating tissue necrosis, accelerating the general skin healing. Allantoin is, most importantly, utilized for its healing capacities for skin acne and rosacea [35]. This excipient can relieve the skin without inducing any irritation. By empowering the regular revival process, allantoin can help to get relieved from skin acne and make a more reliable skin tone. Allantoin also helps the skin to hold the moisture content, the compound additionally uplifts the skin to exfoliate dead skin cells and develop new ones, which helps in the healthy development of skin and averts dull or pallid skin. In case of delicate, dry, or layered skin, utilizing items that contain allantoin may profit it by nourishing, 
securing, and recuperating all in the meantime without inducing any further unfavorable side effects [36].

Cosmetics that contain allantoin can be a beneficial decision with regard to smoothing and mending the defects [24]. Therefore, it is utilized for the treatment of skin bruises, skin sores, lesions, sunburns, scalds, burns, imperfections, spots, skin inflammation and skin emissions, gaps and scraped areas, impetigo, dermatitis, and psoriasis [35].

\section{Porphyra}

Porphyra (Bangiales, Rhodophyta) contains high amount of free amino acids; when introduced in extreme radiations, UV-absorbing secondary metabolites are synthesized, for example, mycosporine-like amino acids. There are just about seven types of Porphyra recognized in India. Among these, Porphyra vietnamensis is increasing more consideration. Conventional Aloe vera gel had low retention capacity over wide UV wavelength (250-400 nm) as contrasted with isolated compound gel, recommending that Porphyra-334 is more potent in nature [22]

\section{Apricot}

Apricot is a magnificent alternative for plastic microbeads [37]. Granules of apricot tenderly shed dead cells of the skin; bring out the natural glow of the skin. It also helps in removing blackheads and prevents the reoccurring of acne [38]. Apricot is utilized in many cosmetics for personal care of skin and body and bath products [37]. There are numerous cosmetics products which utilized apricot as the chief ingredient in their formulations. Topical application of, Apricot Kernel Carrier Oil nourishes dry, delicate skin and treat imperfections, fine lines, blemishes, wrinkles, and different side effects of skin aging [39].

\section{Neem}

Neem obtained from the Azadirachta indica. Neem leaves have awesome advantages for the skin. Neem leaves can be utilized as a face pack that lessens acne caused by microbes. In addition, the face pack of neem leaves has high amount of Vitamin E, will guarantee the skin to hold the moisture and does not dry out. Utilizing steam of neem leaves can help in giving toned skin and expel blackheads [40]. Leaf granules of neem are utilized particularly in herbal face packs. Because of its high antibacterial and antifungal activities, neem extract can be utilized as skin soother in various cosmetic products [41]

\section{Cucumber}

Cucumber plays an imperative part in skin rejuvenation, correction, and protection [42]. Cucumber has antioxidant and anti-inflammatory action. It contains Vitamin E and Vitamin C [43]. Research and clinical examinations demonstrate that topical application of vitamins is advantageous to cure several skin issues and particularly, to avert delay or seize specific age-related degenerative changes, for example, skin desquamation and dryness. Besides, the nature of vitamins has incited their utilization in creams and moisturizers to keep up a delicate and smooth skin by "recharging nature's moisture" [42].

\section{Skin moisturizers}

Coconut oil

Coconut oil is obtained from the Cocos nucifera having family, Arecaceae. Coconut oil contains glycerides of lower chain unsaturated fats. Coconut oil can be used in both solid and liquid forms, as the melting point of an oil is $24-25^{\circ} \mathrm{C}$. Coconut oil has amazing skin smoothening and nourishing property $[4,16]$. Coconut oil has sweet odor excellent penetration capacity, which makes it a powerful moisturizing agent [44]. This oil replenishes the natural oils of your skin and gives a natural glow to your skin [45]. It also helps in slow down the aging effects and keeps your skin hydrated, which makes the skin soft and supple [45]. Furthermore, the utilization of coconut oil is effective and safe when applied as a moisturizer on the skin. The study inferred that utilization of coconut oil is useful for the prevention of protein loss from the wet hair combing over when utilized for $14 \mathrm{~h}[4,16]$.
Jojoba oil

Jojoba oil is obtained from an American shrub called Simmondsia chinensis, family Simmondsiaceae. This oil can be utilized as a moisturizer in beauty care products and as carrier oil due to its characteristic fragrance. Human sebum and jojoba oil are relatively comparable. Human sebum protects and douse the skin of the human body, however cleared by toxins, chemicals, sun, and thus bringing about the fragile skin. This oil has the property of restoring what hair and skin mislay and renews them to their corresponding $\mathrm{pH}$ value so that jojoba oil can be utilized as a natural skin conditioner. Refined type of jojoba oil is fragrance-free which neutralize the necessity of any perfuming vehicle $[16,46]$. Antibacterial action of this oil serves to limit bacterial development; alongside this, it has anti-inflammatory activity. Regular utilization of jojoba oil control acne [46]. Hence, this herb has a great potential to be used as a chief ingredient in herbal cosmetics.

\section{Miscellaneous}

Plant phenolics are dynamic segments of plants, generally utilized as a part of the solution to cure skin diseases when used topically, i.e., to exert anti-inflammatory action, quicken skin wound recuperating, and ensure human skin against injurious impacts of sun oriented radiations. Dermatological or cosmetics formulations consisting phenolic extracts are known for several properties such as chemoprevention, photoprotection, wound healing, anti-aging and so on, yet they could be a two-fold edged sword for human skin, having both defensive and harming activities [47].

\section{Quercetin}

Quercetin has antioxidant and anti-inflammatory activity and works as an effective immunomodulator. Rutin and quercetin were found to provide protection against UVA and UVB radiations when applied topically on the human skin. Thus, they act as a potential excipient in various sunscreen formulations [22]. Quercetin elevates the intracellular oxidation inhibiting property and secures the human keratinocytes from UV radiations (mainly UVA) damage [47].

- Antioxidant activity: Quercetin is likely the dynamic compound that assembles every property fundamental for an effective oxidation inhibiting activity, and it is utilized as a part of nourishing and antiaging formulations [48].

- Protein inhibition: Quercetin, kaempferol, myricetin, morin, and rutin inhibit the 5-lipoxygenase enzyme. Likewise, quercetin inhibits the cyclooxygenase and 12-lipoxygenase enzymes [48].

- Anti-allergic effect: Quercetin, kaempferol, and myricetin inhibit the release of numerous mediators like histamine [48].

Grapes are one of the rich sources of quercetin. The oxidation inhibiting the activity of grape parts, its formulations and by-products are notable, for high radical scavenging activity in relation to Vitamins $\mathrm{E}$ and $\mathrm{C}$ and Trolox, the possibility to repress lipid oxidation in different food and cell models and their chelating properties. When root extract of Vitis vinifera added to cultured typical human keratinocytes, it showed in vitro antioxidant activity. This oxidation inhibiting property was found to be stronger than Vitamins E and C. After 4 weeks of its application, a dermatologic assessment indicated a sign of improvements in the fundamental clinical indications of photoaged skin. Grape-based formulations are productive in improving untimely maturing and could decrease the event of skin disease and defer the procedure of photoaging. The grape treatment has been proposed for the cure of extraneous skin maturing forms. Few conventional items contain pure natural compounds from grape, for example, quercetin, procyanidins, or resveratrol, and melatonin, which are efficient in deferring the onset of an assortment of age-related skin problems [47].

\section{Resveratrol}

Resveratrol is a fat-soluble compound, comes under the classification of polyphenolic compounds known as stilbenes. It is a naturally occurring polyphenolic phytoalexin. Resveratrol was found to mediate anti-inflammatory action, exerts antioxidant activity, and it intervened 
mitigating impacts. Topical utilization of resveratrol SKH-1 bald mice preceding UVB light brought about a huge lessening in UVB-age of $\mathrm{H}_{2} \mathrm{O}_{2}$ and additionally invasion of leukocytes and restraint of skin edema. Long haul thinks about have shown that topical application with resveratrol (both pre- and post-treatment) brings about hindrance of UVB-initiated tumor frequency and postponement in the beginning of skin tumorigenesis [22]. Resveratrol, a unique substrate for tyrosinase with promising corrective points of view is biotransformed by tyrosinase into an oxidized form, which goes about as an intense inhibitor of tyrosinase and was proposed as an added substance in brightening beauty care products, especially with a Morus alba extricate. Oxyresveratrol indicated 32-overlay more grounded inhibitory impacts on mushroom tyrosinase movement than kojic acid [47]. Resveratrol, a normally happening atom found, for example, in monster knotweed, grapes and, thusly, in red wine, has been the subject of serious research as of late. Logical reports are progressively showing the multi-utilitarian advantages of resveratrol. Resveratrol is accounted for as a modulator of hereditary articulation through flag transduction, an inhibitor of provocative middle people, to have phytohormonal benefits, and to lessen the combination of melanin. Such a mix of organic capacities and the corrective impacts makes resveratrol a one of a kind dynamic element for individual care products [49].

\section{Curcumin}

Curcumin also called as diferuloylmethane. Curcumin is obtained through the isolation of turmeric rhizome, which is known as Curcuma longa. It is a yellow, odorless pigment. Curcumin has calming, antioxidant, and antitumoral properties. When curcumin was topically applied on the CD-1 mice epidermis, it has been discovered that curcumin significantly hindered UVA-instigated ornithine decarboxylase action. Curcumin has the ability to scavenge ROS by its inhibitory action. Curcumin can avoid apoptotic changes induced from UV rays in human epidermoid carcinoma A431 cells [22]. While analyzing the defensive impacts of iron, an oxidation inhibiting agent, on the skin, scientists from Unilever and Newcastle University in the U.K found that curcumin showed $8 \%$ protection against UVA rays and $16 \%$ protection against oxidative stress [50]. It can be used as an adjuvant along with nonsteroidal anti-inflammatory drugs in the treatment of osteoarthritis [51]. Turmeric powder with natural products like milk is compelling natural and effective beautifiers in themselves; it makes the skin beautiful and gives a healthy glow. Likewise, they control skin loosing and wrinkle formation on the skin surface, thus help to reestablish and maintain healthy skin. Turmeric can profit various skin problems including dermatitis, psoriasis skin inflammation, and acne. Viable mending properties of turmeric have made it acknowledged after excipient in beauty care products and medications, as the leaf oil of turmeric and concentrate can likewise be utilized as sun blockers [52]

\section{Green and black tea}

Tea (Camellia sinensis) is normally utilized as one of the natural remedies for sunburn. Theobromine and tannic acid are the chief components of tea, which are enabled to remove heat from sunburns. The Chinese prescribe that application of cooled black tea on the skin surface soothe sunburn. Another major component of tea known is catechin, which helps to avert and repair the damage of skin and may even help to counteract radiation and chemical initiated skin malignancies. Various polyphenolic compounds in tea give an indistinguishable defensive impact on the skin as for inner organs. These polyphenolic compounds have been discovered to balance biochemical pathways that are essential for multiplication of cell, provocative reactions, and reactions of tumor promoters. Green tea has been found to provide antioxidant and anti-inflammatory activity in both animal and human skin.

Experimental studies in animals showed evidence that oral or topical application of tea polyphenols, ameliorate unfavorable skin responses following UV radiation exposure, including erythema, skin damage, and lipid peroxidation. Oxidative stress and inflammation seem to play a critical part in the skin maturing procedure, green tea may likewise have anti-aging impacts by diminishing inflammation, irritation, and scavenging free radicals. Scientists have discovered that the principal component in green tea, EGCG, functions admirably as a calming, oxidation inhibiting agent, anti-inflammatory, and sunscreen. Topical application of green tea on human skin gives a photoprotective impact, lessened the quantity of sunburns cells, shielding UV radiation harm to epidermal Langerhans cells, and decreased the DNA harm that occurs from UV radiation. Topical and oral administration of green tea in mice was found to diminish melanoma cell arrangement. Various cosmetics formulations comprising tea concentrates or phenols have not been tested in controlled clinical trials, but rather these substances have demonstrated convincing confirmation for anti-inflammatory, antioxidant, and anticarcinogenic properties.

\section{A. vera}

It is a herbal plant obtained from aloe barbadensis, belonging to the family Lililiaceae. It comprises various amino acids such as leucine, isoleucine, and saponin glycosides that provide cleanser action, folic acid, Vitamins B, A, E, C, and B12 give antioxidant action. It is utilized as a principal excipient in different cosmeceuticals products as it is having bacteriostatic and antiphlogistic activity $[6,8]$. A. vera can be classified in the following fundamental zones: Anthraquinones, amino acids, lignin, catalysts, minerals, mono- and polysaccharides, saponins, vitamins, salicylic acid, and sterols. A. vera improved fibroblast cell structure, as well as quickened the collagen creation process [22]. A. vera forms the coat on the surface of skin and shields the skin from harmful UV radiations of the sun. It diminishes the wrinkles and aides in the lightning of the dark spots and imperfections. $[16,53]$. Dermatologists prescribe $A$. vera to cure sunburn, lighten stretch marks and sun tan as $A$. vera have the healing property in the skin cells up to the epithelial level [53,54].

The herbal plant $A$. vera has been scientifically demonstrated for all types of burn, be it solar, thermal, or radiation-based. It has additionally been shown that $A$. vera has a prophylactic impact if utilized prior, during and after these skin harming conditions. Clearly, this herbal plant is essentially utilized for its cooling and calming property. However, this herbal excipient is futile if utilized in half proportion, and it is prescribed that it is utilized at $100 \%$ to make certain of any gainful impact. The anthraquinones, mannose-6-phosphate, and polysaccharides all provide synergistic action to the benefits of this herbal excipient. A. vera is a remarkably powerful moisturizer and recuperating operator for both animal and human skin [22].

\section{Ubtan/mask for skin}

Ubtan is a herbal formulation, being utilized customarily in India and other Asian countries for its cosmetic benefits. Tyrosinase or polyphenol oxidase is a multi-functional copper comprising enzyme, in charge of the amalgamation of melanin inside the melanocytes. Two noteworthy activities of this compound are the hydroxylation of L-tyrosine to 3,4-dihydroxyphenylalanine (L-DOPA) (monophenolase) and subsequent oxidation of L-DOPA to dopaquinone (diphenolase). Dopaquinone is used to produce melanin through the inter-mediated biosynthetic pathway. It is mixture heterogeneous biopolymers directing the color of skin. It has a defensive role in retaining UV radiations from the sun and scavenging ROS from skin. Hyperpigmentation can occur as a result of the accumulation of overabundance amount of melanin in the skin. Melasma is the most widely recognized hyper-melanosis disease in the Asian locale among individuals who live inside the region of intense sun exposure. Melanin associated skin darkening can be treated with tyrosinase inhibitors. Therefore, these tyrosinase inhibitors are valuable therapeutic agents for skin lightning

Melanin inhibitor perspective of some mercury salts (organic and inorganic) makes them broadly utilized excipient of skin brightening formulations. They penetrate through the skin and produce destructive symptoms in the body, for example, skin discoloration, kidney harm, scarring, and unfavorably susceptible response, and additionally in the skin, lose its protection from bacterial and parasitic diseases. In India, $61 \%$ of the dermatology products comprises skin lightening items. Many tests have been done to determine the heavy metals such 
Table 1: Marketed products of herbal skin cosmetics

\begin{tabular}{|c|c|c|c|}
\hline Products & $\begin{array}{l}\text { Active } \\
\text { ingredient }\end{array}$ & Ingredients & Reference \\
\hline Deep cleansing apricot face wash (Himalaya) & Apricot & Apricot, Aloe vera, Neem, Red lentin & {$[38]$} \\
\hline Fairness kesar face wash (Himalaya) & Kesar & Kesar, cucumber, pomegranate, mint & [57] \\
\hline Tea tree face wash (Ayur) & Tea Tree & Tea tree oil, citric acid, turmeric & [58] \\
\hline Lemon honey face wash (Patanjali) & Lemon, honey & Lemon, honey, Tulsi & [59] \\
\hline Purifying neem scrub (Himalaya) & Neem & Neem, apricot & {$[60]$} \\
\hline Aloe vera moisturizing cream (Patanjali) & Aloe vera & Aloe vera, Shea butter, coconut oil, wheat germ oil & [61] \\
\hline Herbal moisturizer (Ayur) & Wheat germ oil & Olive oil, turmeric extract, wheat germ oil & {$[58]$} \\
\hline Purifying neem pack (Himalaya) & Neem & Neem, tumeric, Fuller's Earth & [63] \\
\hline Apricot and honey peel off mask (Ayur) & Apricot, honey & Apricot, honey & [58] \\
\hline
\end{tabular}

Table 2: Patents on herbal cosmetics

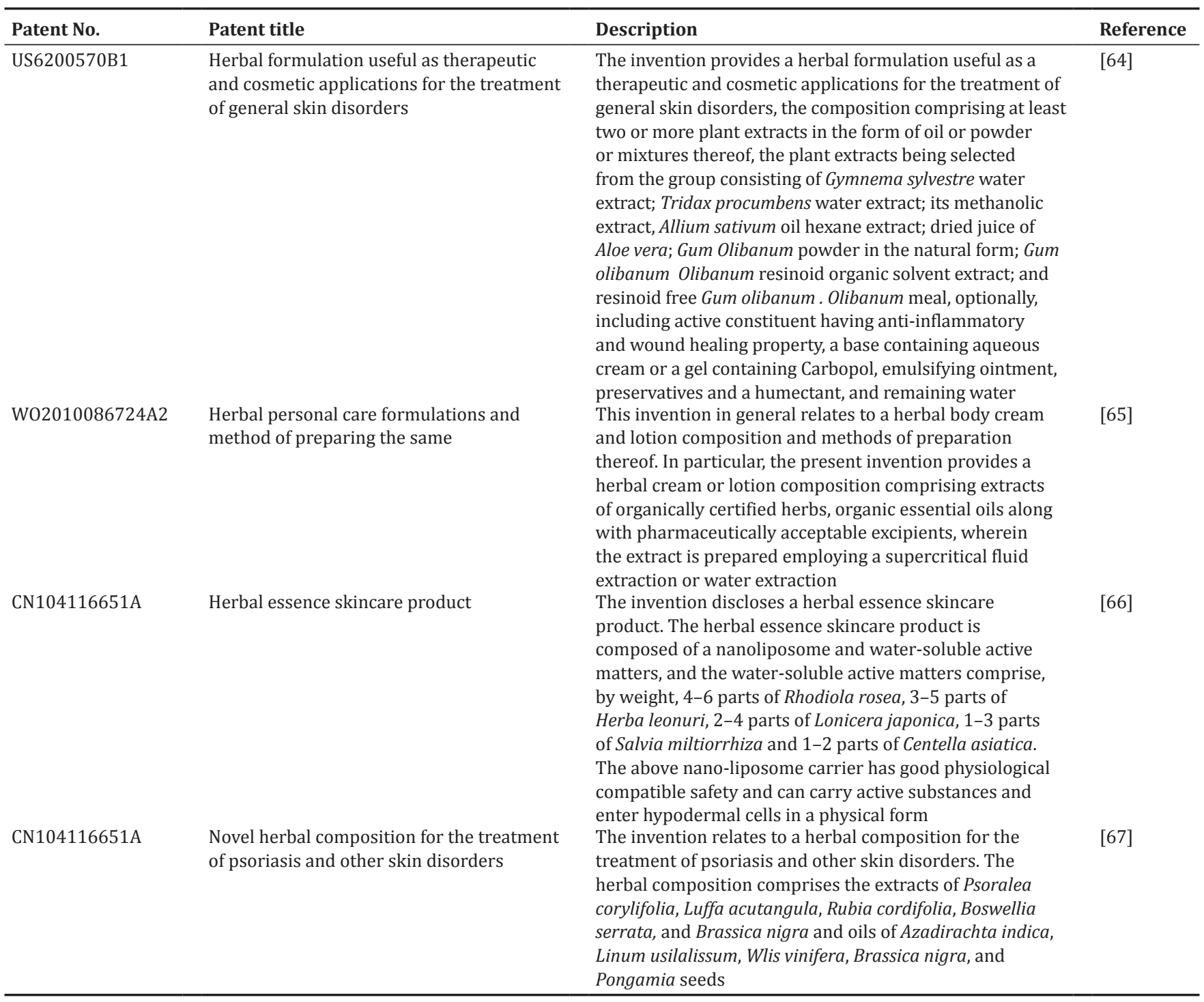

as $\mathrm{Pb}, \mathrm{Hg}$, As, and $\mathrm{Cd}$ and trace components in natural cosmeceuticals preparations utilized in the Indian cosmetic market. Most of them contain heavy metals over as far as possible.

Ubtan is a well-known beautifying and improving cosmeceuticals in India developed from traditional information. The scientific standard of Ubtan was created in view of its therapeutic and phytochemical claim. Further, it was investigated for its activity against oxidant and tyrosinase movement.
Furthermore, heavy metals were evaluated by the (i.e., Atomic Absorption Spectroscopy) for its appraisal of security for topical application [55].

\section{MARKETED PRODUCTS}

There are various products that are available in the market, which is based on herbal ingredients. The market size is expanding for herbal cosmetics. However, there is always need for regulatory control for 
better utilization and safety of end users. Some of the marketed formulations of herbal cosmetics are enlisted in Table 1. The some of the related patents are also enlisted in Table 2.

\section{FUTURE PROSPECTS}

Further, the effectiveness of herbal skin cosmetics can be enhanced with the use of nanoformulations for skincare cosmetics. Nano liposomes, niosomes, etc., have been found to have a greater affinity to skin, enhanced penetration, and stability of herbal ingredients. Nanotechnology-based cosmetic preparations have a wide scope for herbal cosmetics also in the near future.

\section{CONCLUSION}

Throughout the most recent couple of years, countless evidence has developed, demonstrating that chemically different classes of naturally occurring substances are potent in the treatment of several dermatological disorders caused by UV radiation exposure. Flavonoids, terpenes, polyphenols, alkaloids, and catechins are chemical classes that have been focused for photochemopreventive property and resveratrol, curcumin, quercetin and caffeic acid are a couple of examples of such compounds. These antioxidants have been suggested as a beneficial strategy for diminishing the UV-radiation intervened oxidative harm to the skin. For instance, the topical application or oral administration of plant concentrates, for example, black and green tea, A. vera, coffee, apricot, coconut oil, cucumber, and quercetin is speculated to be defensive against premature aging, UV-initiated erythema and radiation prompted tumor.

Plants and their extracts, by-products and products, have been efficiently utilized as a part of herbal cosmeceuticals in Sri Lanka for curing ailments for more than 1000 years. More than 1400 plants are utilized in the native flora of Sri Lanka as a part of indigenous medication, and the review of literature demonstrates that ample numbers of plants are extensively used for the treatment of different dermatological conditions and also to improve or lighten the skin complexion [56]. These herbal excipients appear to be encouraging, beneficial and free from adverse effects, despite the fact that their actual impacts are not logically demonstrated, in this way facilitate examinations ought to be performed to evaluate the clinical advantages. However, bunch of logical confirmations is available on bioactivity investigations of plants having therapeutic value that could lead toward the formation of herbal cosmetics. The study revealed that green and black tea has photoprotective properties. The present examination has concentrated on the assessment of antioxidant and sun screening activity of eleven therapeutic plants that have been widely used in Sri Lanka as dermatological therapeutics for treating various dermatological conditions and for lightening complexion. Consequently, the pharmacological utilization of these herbal plants could be rationalized. These herbal plants can be effectively used as dermatological therapeutics for treating various dermal disorders such as eczema, psoriasis, skin inflammation, aging, irritation and as principal herbal excipients in various cosmeceuticals.

\section{ACKNOWLEDGMENTS}

The authors are thankful to Dr. Madhu Chitkara, Vice-Chancellor, Chitkara University; Dr. Ashok Chitkara, Chancellor, Chitkara University; Dr. Sandeep Arora, Director, Chitkara College of Pharmacy for providing necessary facilities and support.

\section{AUTHORS' CONTRIBUTIONS}

Riya Arora has compiled the whole manuscript, Dr. Geeta Aggarwal has generated the idea, and Dr. Gitika Arora helped in the compilation of manuscript and both provided necessary guidance while compilation and Dr. Manju Nagpal remove the plagiarism and routine check while drafting the manuscript and provided all full-text literature.

\section{CONFLICTS OF INTEREST}

The authors declared that they have no conflicts of interest.

\section{REFERENCES}

1. Patkar KB. Herbal cosmetics in ancient India. Indian J Plast Surg 2008;41:S134-7.

2. Joshi B. Herbal Cosmetics: A Safe and Effective Approach. Pharmatutor. Available from: https://www.pharmatutor.org/articles/ herbal-cosmetics-used-for-skin-hair-care. [Last accessed on $2018 \mathrm{Aug}$ ].

3. Kapoor VP. Herbal cosmetics for skin and hair care. Nat Prod Radiance 2005;4:306-14.

4. Gediya SK, Mistry RB, Patel UK, Blessy M, Jain HN. Herbal plants: Used as a cosmetics. J Nat Prod Plant Resour 2011;1:24-32.

5. Edward F. 6 Reasons to Use Organic Makeup. Global Healing Center; 2014. Available from: https://www.globalhealingcenter.com/naturalhealth/6-reasons-to-use-organic-makeup. [Last accessed on $2018 \mathrm{Oct}$ ].

6. Bijauliya RK, Alok S, Kumar M, Chanchal DK, Yadav S. A comprehensive review on herbal cosmetics. Int J Pharm Sci Res 2017;8:4930-49.

7. Castanet J, Ortonne JP. Pigmentary changes in aged and photoaged skin. Arch Dermatol 1997;133:1296-9.

8. Chung JH, Hanft VN, Kang S. Aging and photoaging. J Am Acad Dermatol 2003;49:690-7.

9. Rabe JH, Mamelak AJ, McElgunn PJ, Morison WL, Sauder DN. Photoaging: Mechanisms and repair. J Am Acad Dermatol 2006;55:1-9.

10. Chowdhury R. Anti-Ageing Herbs for Beautiful Skin. News Boldsky Limitless Living; 2017.

11. Binic I, Lazarevic V, Ljubenovic M, Mojsa J, Sokolovic D. Skin ageing: Natural weapons and strategies. Evid Based Complement Altern Med 2013;2013:1-10.

12. Shin S, Lee JA, Son D, Park D, Jung E. Anti-skin-aging activity of a standardized extract from Panax ginseng leaves in vitro and in human volunteer. Cosmetics 2017;4:18.

13. Hwang E, Park SY, Yin CS, Kim HT, Kim YM, Yi TH, et al. Antiaging effects of the mixture of Panax ginseng and Crataegus pinnatifida in human dermal fibroblasts and healthy human skin. J Ginseng Res 2017;41:69-77.

14. Cho S, Won $\mathrm{CH}$. Red Ginseng and Its effect on aging skin. FYI Living 2009; 12:1252-9.

15. Challoner NI, Chahal SP, Jones RT. Cosmetic proteins for skin care: An investigation into the use of proteins as moisturizing and tightening agents. Cosmet Toiletries 1997;112:51-63.

16. Joshi LS, Pawar HA. Herbal cosmetics and cosmeceuticals: An overview. Nat Prod Chem Res 2015;3:2.

17. Gopi R, Jaleel CA, Sairam R, Lakshmanan GM, Gomathinayagam M, Panneerselvam R. Differential effects of hexaconazole and paclobutrazol on biomass, electrolyte leakage, lipid peroxidation and antioxidant potential of Daucus carota L. Colloids and Surfaces B: Biointerfaces. 2007;60:180-6.

18. Danielpour P, Layke J. Ginkgo biloba: Ancient Herb with Amazing Anti-Aging Powers. Beverly Hills MD; 2016. Available from: https:// beverlyhillsmd.com/ginkgo-biloba-skincare/ [Last accessed on $2018 \mathrm{Aug}$ ].

19. Singh M. The Role of Antioxidants in Dermtological and Cosmetic Formulas. Available from: https://www.happi.com/issues/2013-12/ view_features/the-role-of-antioxidants-in-dermtological-cosmeticformulas. [Last accessed on 2018 Oct].

20. Ribeiro A, Estanqueiro M, Oliveira M, Lobo JS. Main benefits and applicability of plant extracts in skin care products. Cosmetics 2015;2:48-65.

21. Freeman BB, Sandhu A, Edirisinghe I. Nutraceuticals Efficacy, Safety and Toxicity. Amsterdam: Academic Press; 2016. p. 489-500.

22. Korać RR, Khambholja KM. Potential of herbs in skin protection from ultraviolet radiation. Pharmacogn Rev 2011;5:164-73.

23. McDougall A. From fighting cancer to fighting wrinkles: Anthocyanins launched with cosmetic benefits. APAC 2011. Available from: https:// www.cosmeticsdesign-europe.com/Article/2011/10/05/From-fightingcancer-to-fighting-wrinkles-anthocyanins-launched-with-cosmeticbenefits [Last accessed on 2018 Aug].

24. Zouboulis, C. Retinoids retinol skincare benefits antioxidants and antiaging l'oreal Paris. Skin Pharmacol Physiol 2001;14:303-15.

25. Saewan N, Jimtaisong A. Photoprotection of natural flavonoids. J Appl Pharm Sci 2013;3:129-41.

26. Bird K. Natural Ingredients Protect Against UV. Available from: https:// www.cosmeticsdesign.com/Article/2007/12/20/Natural-ingredientsprotect-against-UV. [Last accessed on 2018 Sep]. 
27. Kaur AK, Wahi AK, Brijesh K, Bhandari A, Prasad N. Milk thistle (Silybum marianum): A review. Int J Pharm Res Dev 2011;3:1-10.

28. Hung CF, Lin YK, Zhang LW, Chang CH, Fang JY. Topical delivery of silymarin constituents via the skin route. Acta Pharmacol Sin 2010;31:118-26

29. Marta. Plant Extracts that Provide Sun Protection. Website. Truth in Aging; 2009. Available from: https://www.truthinaging.com/review/ plant-extracts-that-provide-sun-protection. [Last accessed on $2018 \mathrm{Aug}$ ].

30. Telang PS. Vitamin C in dermatology. Indian Dermatol 2013;4:143.

31. Akruti. 10 Amazing Benefits of Walnut Oil for Skin, Hair and Health. Ingredients and Uses; 2017. Available from: https://www.stylecraze. com/articles/amazing-benefits-of-walnut-oil-for-skin-hair-andhealth/\#gref. [Last accessed on 2018 Oct].

32. Available from: https://www.truthinaging.com. [Last accessed on 2018 Sep]

33. Fatima A, Alok S, Agarwal P, Singh PP, Verma A. Benefits of herbal extracts in cosmetics: A review. Int J Pharm Sci Res 2013;4:3746-60.

34. Patterson S. 10 Reasons to Put Tea Tree Oil on Your Skin: Beat Acne, Eczema and More. Natural Living Ideas; 2017. Availablr from: https:// www.naturallivingideas.com/tea-tree-oil-for-skin/. [Last accessed on 2018 Sep].

35. Carosi S. Allantoin Skin Treatment Introduction. The Essence of Mineral Makeup. Available from: https://www.essence-of-mineralmakeup.com/allantoin.html. [Last accessed on 2018 Sep]

36. Available from: https://www.thedermreview.com/allantoin. [Last accessed on $2018 \mathrm{Sep}]$.

37. Available from: http://www.compomat.com/cosmetics-gradeingredients. [Last accessed on $2018 \mathrm{Sep}$ ].

38. Available from: http://www.himalayawellness.com/products/personalcare/ deep-cleansing-apricot-face-fash.htm. [Last accessed on 2018 Sep]

39. Apricot Kernel Oil A Light and Quick Absorbing Oil-Skin and Hair Care. New Directions Aromatics Blog; 2017. Availablr from: https:// www.newdirectionsaromatics.com/blog/products/all-about-apricotkernel-carrier-oils.html. [Last accessed on 2018 Sep]

40. Tadimalla RT. Neem: Benefits, Uses, History, and Side Effects. Stylecraze; 2018. Available from: https:/www.medicalnewstoday.com/ articles/325048.php. [Last accessed on 2018 Sep].

41. Neem Cosmetics, Neem Cosmetics Products, Neem Used for Cosmetics, Neem Herbal Cosmetics. Neem Products. Available from: http://www.neem-products.com/neem-cosmetics.html . [Last accessed on 2018 Sep].

42. Idson B. Cucumber. Centerchem; 1993. Available from: https://www. scribd.com/document/359324229/Cucumber-Extract. [Last accessed on 2018 Sep].

43. Szalay B. Cucumbers: Health Benefits and Nutrition Facts. Live Science; 2017. Available from: https://www.livescience.com/51000cucumber-nutrition.html . [Last accessed on 2018 Oct].

44. Kellogg, K. 30 Coconut Oil Uses for Beauty: Unexpected Hair and Skin Benefits. Allure. Magazine; 2017.

45. Keong L. 15 Surprising Beauty Benefits of Coconut Oil. Magazine. Marie Claire; 2016.

46. Patterson S. 12 Surprising Benefits of Jojoba Oil for Beautiful Skin and Hair. Natural Living Ideas; 2015. Available from: https://www. naturallivingideas.com/jojoba-oil-benefits-for-skin-hair/ [Last accessed on 2018 Oct].
47. Soto M, Falqué E, Domínguez H. Relevance of natural phenolics from grape and derivative products in the formulation of cosmetics. Cosmetics 2015;2:259-76.

48. Benaiges A, Guillen P. Botanical extracts. In: Analysis of Cosmetic Products. Amsterdam: Elsevier B.V.; 2005. p. 345-63. DOI: 10.1016/ B978-044452260-3/50044-9.

49. Beck M, Den Brave K, Flores-Candia JL, Lu Y. Cosmetic Composition for Skin Whitening Comprising Resveratrol. United States Patent Application US 13/884,397. Inventors; DSM IP Assets BV; 2013.

50. Faseb. Curcumin and Skin Health. Natural Products Insider; 2015. Available from: https://www.naturalproductsinsider.com/beauty/ curcumin-and-skin-health. [Last accessed on 2018 Sep].

51. Sulthana N, Vijaya K. Prevention of peptic ulcers by curcumin in chemically induced osteoarthritis. Int J Pharm Pharm Sci 2018;10:29-34.

52. Available from: http://www.turmeric.co.in/about us.html. [Last accessed on 2018 Sep].

53. Malik K. 6 Amazing Benefits of Aloe vera for Hair, Skin and WeightLoss. NDTV Food News; 2018.

54. Paul SU, Dutta SO, Chaudhuri TK, Bhattacharjee SO. Antiinflammatory and protective properties of Aloe vera leaf crude gel in carrageenan induced acute inflammatory rat models. Int J Pharm Pharm Sci 2014;6:368-71

55. Biswas R, Mukherjee PK, Kar A, Bahadur S, Harwansh RK, Biswas $\mathrm{S}$, et al. Evaluation of ubtan a traditional Indian skin care formulation. J Ethnopharmacol 2016;192:283-91

56. Napagoda MT, Malkanthi BM, Abayawardana SA, Qader MM, Jayasinghe L. Photoprotective potential in some medicinal plants used to treat skin diseases in Sri Lanka. BMC Complement Altern Med 2016;16:479.

57. Available from: http://www.himalayawellness.com/products/ personalcare/fairness-kesar-face-wash.html.

58. Available from: http://www.ayurherbals.com/facecare.html.

59. Available from: https://www.beautifulhameshablog.com/patanjalilemon-honey-face-wash-review. [Last accessed on 2018 Oct].

60. Available from: http://www.himalayawellness.com/products/personalcare/ purifying-neem-scrub.html. [Last accessed on 2018 Oct].

61. Available from: https://www.beautifulhameshablog.com/patanjalialoe-vera-moisturizing-cream-review. [Last accessed on 2018 Nov]

62. Available from: http://www.himalayawellness.com/products/personalcare/ nourishing-skin-cream.html. [Last accessed on 2018 Nov]

63. Available from: http://www.himalayawellness.com/products/personalcare/ purifying-neem-pack.html. [Last accessed on 2018 Nov].

64. Ramakrishna S, Sitaramam BS, Diwan PV, Raghavan KV, Herbal Formulation Useful for Treatment of Skin Disorders. United States Patent US 6,383,495. Inventors; Council of Scientific, Industrial Research CSIR; 2002.

65. Surianarayanan R, Manral R, Babu UV, Saxena E, Mahendra C. Herbal Personal Care Formulations and Method of Preparing the Same. United States patent US 9, 358, 263; 2016 .

66. Herbal Essence Skin Care Product. CN104116651A; 2014.

67. Babu UV, Mishra B. Novel Herbal Composition for Treatment of Psoriasis and other Skin Disorders. United States Patent Application US 12/981,742. Inventors; Himalaya Global Holdings Ltd.; 2011 